\title{
The Strong Thirteen Spheres Problem
}

\author{
Oleg R. Musin • Alexey S. Tarasov
}

Received: 21 July 2010 / Revised: 27 July 2011 / Accepted: 16 October 2011 /

Published online: 29 February 2012

(C) Springer Science+Business Media, LLC 2012

\begin{abstract}
The thirteen spheres problem asks if 13 equal-size non-overlapping spheres in three dimensions can simultaneously touch another sphere of the same size. This problem was the subject of the famous discussion between Isaac Newton and David Gregory in 1694. The problem was solved by Schütte and van der Waerden only in 1953.

A natural extension of this problem is the strong thirteen-sphere problem (or the Tammes problem for 13 points), which calls for finding the maximum radius of and an arrangement for 13 equal-size non-overlapping spheres touching the unit sphere. In this paper, we give a solution of this long-standing open problem in geometry. Our computer-assisted proof is based on an enumeration of irreducible graphs.
\end{abstract}

\section{Introduction}

\subsection{The Thirteen-Sphere Problem}

The kissing number $k(n)$ is the highest number of equal non-overlapping spheres in $\mathbb{R}^{n}$ that touch another sphere of the same size. In three dimensions, the kissing number problem is how many white billiard balls can kiss (touch) a black ball.

The most symmetrical configuration, 12 balls around one ball, is achieved if the 12 balls are placed at positions corresponding to the vertices of a regular icosahedron

O.R. Musin

Department of Mathematics, University of Texas at Brownsville, 80 Fort Brown, Brownsville, TX 78520, USA

e-mail: oleg.musin@utb.edu

A.S. Tarasov ( $\varangle)$

Institute for System Analysis, Russian Academy of Science, 9 Pr. 60-letiya Oktyabrya, Moscow, Russia

e-mail: tarasov.alexey@gmail.com 
concentric with the central ball. However, these 12 outer balls do not kiss each other and may all be moved freely. The space between the balls leads to a question: If you moved all of them to one side, would a 13th ball fit?

This problem was the subject of the famous discussion between Isaac Newton and David Gregory in 1694 (May 4, 1694; see [29] for details of this discussion). Most reports say that Newton believed the answer was 12 balls, while Gregory thought that 13 might be possible. However, Casselman [10] found some puzzling features in this story.

This problem is often called the thirteen-sphere problem. Hoppe [15] thought he had solved the problem (1874). But he made a mistake, and an analysis of this mistake was published by Hales in 1994 [14] (see also [29]). The problem was finally solved by Schütte and van der Waerden in 1953 [28]. A subsequent two-page sketch of an elegant proof was given by Leech [17] in 1956. Leech's proof was presented in the first edition of the well-known book by Aigner and Ziegler [1]; the authors removed this chapter from the second edition because a complete proof would have to include much spherical trigonometry.

The thirteen-sphere problem continues to be of interest, and new proofs have been published in the last several years by Hsiang [16], Maehara [19, 20] (this proof is based on Leech's proof), Böröczky [5], Anstreicher [2], and Musin [21].

Note that, for $n>3$, the kissing number problem is solved only for $n=8,24$ [18, 23], and for $n=4$ [22] (see [25] for a beautiful exposition of this problem).

\subsection{The Tammes Problem}

If $N$ unit spheres kiss the unit sphere in $\mathbb{R}^{n}$, then the set of kissing points is an arrangement on the central sphere such that the (Euclidean) distance between any two points is at least 1 . This allows us to state the kissing number problem in another way: How many points can be placed on the surface of $\mathbb{S}^{n-1}$ so that the angular separation between any two points is at least $60^{\circ}$ ?

This leads to an important generalization: a finite subset $X$ of $\mathbb{S}^{n-1}$ is called a spherical $\psi$-code if for every pair $(x, y)$ of $X$ with $x \neq y$ its angular distance $\operatorname{dist}(x, y)$ is at least $\psi$.

Let $X$ be a finite subset of $\mathbb{S}^{2}$. Denote

$$
\psi(X):=\min _{x, y \in X}\{\operatorname{dist}(x, y)\}, \quad \text { where } x \neq y .
$$

Then $X$ is a spherical $\psi(X)$-code.

Denote by $d_{N}$ the largest angular separation $\psi(X)$ with $|X|=N$ that can be attained in $\mathbb{S}^{2}$, i.e.

$$
d_{N}:=\max _{X \subset \mathbb{S}^{2}}\{\psi(X)\}, \quad \text { where }|X|=N .
$$

In other words, we ask: how are $N$ congruent, non-overlapping circles distributed on the sphere when the common radius of the circles has to be as large as possible?

This question, also known as the problem of the "inimical dictators", get be put this way where should $N$ dictators build their palaces on a planet so as to be as 
Fig. 1 An arrangement of 13 points $P_{13}$ and its contact graph $\Gamma_{13}$ with $\psi\left(P_{13}\right) \approx 57.1367^{\circ}$
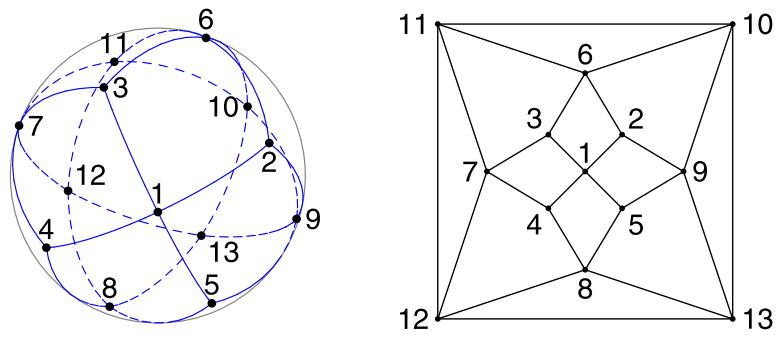

far away from each other as possible? The problem was first asked by the Dutch botanist Tammes [30] (see [8, Sect. 1.6: Problem 6]), who was led to this problem by examining the distribution of openings on the pollen grains of different flowers.

The Tammes problem is presently solved only for several values of $N$ : for $N=$ 3, 4, 6, 12 by L. Fejes Tóth [12]; for $N=5,7,8,9$ by Schütte and van der Waerden [27]; for $N=10,11$ by Danzer [11] (for $N=11$ see also Böröczky [4]); and for $N=24$ by Robinson [26].

\subsection{The Tammes Problem for $N=13$}

The first unsolved case of the Tammes problem is $N=13$, which is particularly interesting because of its relation to the kissing problem and the Kepler conjecture $[6,13,29]$.

Actually, this problem is equivalent to the strong thirteen-sphere problem, which seeks to find the maximum radius of and an arrangement for 13 equal-size nonoverlapping spheres in $\mathbb{R}^{3}$ touching the unit sphere.

It is clear that the equality $k(3)=12$ implies $d_{13}<60^{\circ}$. Böröczky and Szabó [6] proved that $d_{13}<58.7^{\circ}$. Recently Bachoc and Vallentin [3] have shown that $d_{13}<58.5^{\circ}$.

We note that there is an arrangement of 13 points on $\mathbb{S}^{2}$ such that the distance between any two points of the arrangement is at least $57.1367^{\circ}$ (see [13, Chap. VI, Sect. 4]). This arrangement is shown in Fig. 1.

Remark Denote the constant $\psi\left(P_{13}\right)$ by $\delta_{13}$. The value $d=\delta_{13}$ can be found analytically. Indeed, we have (see for notation and functions Fig. 9 and Sect. 3) $u_{0}+2 u_{13}+u_{2}=2 \pi$, where $u_{2}=\pi / 2, a:=u_{0}=\alpha(d), u_{13}=\rho\left(u_{9}, d\right), u_{9}=$ $2 \pi-2 u_{5}, u_{5}=\rho\left(u_{2}, d\right)$. This yields

$$
2 \tan \left(\frac{3 \pi}{8}-\frac{a}{4}\right)=\frac{1-2 \cos a}{\cos ^{2} a}, \quad \cos d=\frac{\cos a}{1-\cos a} .
$$

Thus, we have $a_{13}:=\alpha\left(\delta_{13}\right) \approx 69.4051^{\circ}$ and $\delta_{13} \approx 57.1367^{\circ}$.

\section{Main Theorem}

In this paper, we present a solution of the Tammes problem for $N=13$.

Theorem 1 The arrangement of 13 points in $\mathbb{S}^{2}$ which is shown in Fig. 1 is the best possible; the maximal arrangement is unique up to isometry, and $d_{13}=\delta_{13}$. 
Fig. 2 Danzer's flip
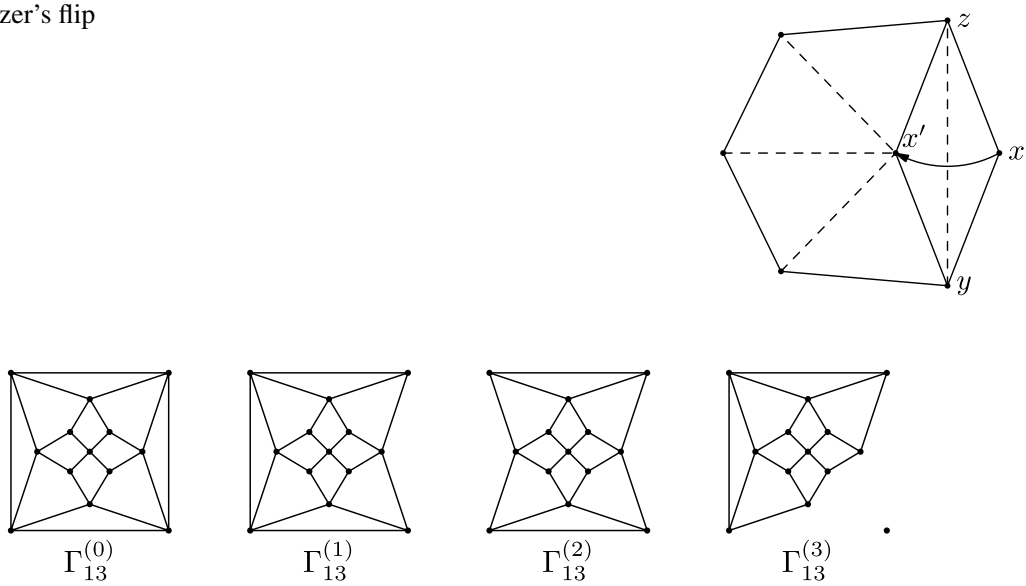

Fig. 3 Graphs $\Gamma_{13}^{(i)}$

\subsection{Basic Definitions}

Contact graphs. Let $X$ be a finite set in $\mathbb{S}^{2}$. The contact graph $\operatorname{CG}(X)$ is the graph with vertices in $X$ and edges $(x, y), x, y \in X$ such that $\operatorname{dist}(x, y)=\psi(X)$.

Shift of a single vertex. Let $X$ be a finite set in $\mathbb{S}^{2}$. Let $x \in X$ be a vertex of $\operatorname{CG}(X)$ with $\operatorname{deg}(x)>0$, i.e. there is $y \in X$ such that $\operatorname{dist}(x, y)=\psi(X)$. We say that there exists a shift of $x$ if $x$ can be slightly shifted to $x^{\prime} \operatorname{such}$ that $\operatorname{dist}\left(x^{\prime}, X \backslash\{x\}\right)>\psi(X)$.

Danzer's flip. Danzer [11, Sect. 1] defined the following flip. Let $x, y, z$ be vertices of $\operatorname{CG}(X)$ with $\operatorname{dist}(x, y)=\operatorname{dist}(x, z)=\psi(X)$. We say that $x$ is flipped over $y z$ if $x$ is replaced by its mirror image $x^{\prime}$ relative to the great circle $y z$ (see Fig. 2). We say that this flip is Danzer's flip if $\operatorname{dist}\left(x^{\prime}, X \backslash\{x, y, z\}\right)>\psi(X)$.

Irreducible graphs. We say that the graph $\mathrm{CG}(X)$ is irreducible ${ }^{1}$ (or jammed) if there are neither Danzer's flips nor shifts of vertices.

$P_{13}$ and $\Gamma_{13}$. Denote by $P_{13}$ the arrangement of 13 points in Fig. 1 . Let $\Gamma_{13}:=$ $\mathrm{CG}\left(P_{13}\right)$. It is not hard to see that the graph $\Gamma_{13}$ is irreducible.

Maximal graphs $G_{13}$. Let $X$ be a subset of $\mathbb{S}^{2}$ with $|X|=13$ and $\psi(X)=d_{13}$. Denote by $G_{13}$ the graph $\operatorname{CG}(X)$. Actually, this definition does not assume that $G_{13}$ is unique. We use this designation for some $\mathrm{CG}(X)$ with $\psi(X)=d_{13}$.

Graphs $\Gamma_{13}^{(i)}$. Let us define four planar graphs $\Gamma_{13}^{(i)}$ (see Fig. 3), where $i=0,1,2,3$, and $\Gamma_{13}^{(0)}:=\Gamma_{13}$. Note that $\Gamma_{13}^{(i)}, i>0$, is obtained from $\Gamma_{13}$ by removing certain edges.

\footnotetext{
${ }^{1}$ This terminology was used by Schütte-van der Waerden [27, 28], Fejes Tóth [13], and Danzer [11].
} 


\subsection{Main Lemmas}

Lemma $1 G_{13}$ is isomorphic to $\Gamma_{13}^{(i)}$ with $i=0,1,2$, or 3 .

Lemma $2 G_{13}$ is isomorphic to $\Gamma_{13}^{(0)}$ and $d_{13}=\delta_{13} \approx 57.1367^{\circ}$.

It is clear that Lemma 2 yields Theorem 1 . Now our goal is to prove these lemmas.

\section{Properties of $G_{13}$}

\subsection{Combinatorial Properties of $G_{13}$}

Proposition 3.1 Let $X$ be a finite set in $\mathbb{S}^{2}$. Then $\mathrm{CG}(X)$ is a planar graph.

Proof Let $a, b, x, y \in X$ with $\operatorname{dist}(a, b)=\operatorname{dist}(x, y)=\psi(X)$. Then the shortest arcs $a b$ and $x y$ do not intersect. Otherwise, the length of at least one of the arcs $a x, a y, b x, b y$ has to be less than $\psi(X)$. This leads to planarity of $\mathrm{CG}(X)$.

The following three propositions are proved in [11] (also see [13, Chap. VI, 6, 7]).

Proposition 3.2 Let $X$ be a subset of $\mathbb{S}^{2}$ with $|X|=N$ and $\psi(X)=d_{N}$. Then for $N>6$ the graph $\mathrm{CG}(X)$ is irreducible.

Proposition 3.3 Let $X \subset \mathbb{S}^{2}$. If the graph $\mathrm{CG}(X)$ is irreducible, then the degrees of its vertices can take only the values 0 (isolated vertices), 3, 4, or 5.

Proposition 3.4 Let $X \subset \mathbb{S}^{2}$ with $|X|=N$. If the graph $\mathrm{CG}(X)$ is irreducible, then its faces are polygons with at most $\left\lfloor 2 \pi / d_{N}\right\rfloor$ vertices.

Böröczky and Szabó [6, Lemmas 8 and 9(iii)] considered isolated vertices in irreducible graphs with 13 vertices.

Proposition 3.5 Let $X \subset \mathbb{S}^{2}$ with $|X|=13$. Let the graph $\mathrm{CG}(X)$ be irreducible. If $\mathrm{CG}(X)$ contains an isolated vertex, then it lies in the interior of a hexagon of $\mathrm{CG}(X)$, and this hexagon cannot contain other vertices of $\mathrm{CG}(X)$.

Combining these propositions, we obtain the following combinatorial properties of $G_{13}$.

\section{Corollary 3.1}

1. $G_{13}$ is a planar graph;

2. any vertex of $G_{13}$ is of degree $0,3,4$, or 5 ;

3. any face of $G_{13}$ is a polygon with three, four, five or six vertices;

4. if $G_{13}$ contains an isolated vertex $v$, then $v$ lies in a hexagonal face. Moreover, a hexagonal face of $G_{13}$ cannot contain two or more isolated vertices. 


\subsection{Geometric Properties of $G_{13}$}

Let $X \subset \mathbb{S}^{2}$ with $|X|=13$. Let the graph $\mathrm{CG}(X)$ be irreducible. Note that all faces of $\mathrm{CG}(X)$ are convex polygons. (Otherwise, a "concave" vertex of a polygon $P$ can be shifted to the interior of $P$.) Then the faces of the graph $\operatorname{CG}(X)$ in $\mathbb{S}^{2}$ are regular triangles, rhombi, convex equilateral pentagons, and convex equilateral hexagons. Polygons with more than six vertices cannot occur. Note that the triangles, rhombi, or pentagons of $\mathrm{CG}(X)$ cannot contain isolated vertices in their interiors. The lengths of all edges of $\mathrm{CG}(X)$ equal $\psi(X)$.

Consider as parameters (variables) of $\operatorname{CG}(X)$ in $\mathbb{S}^{2}$ the set of all angles $u_{i}$ of its faces, and set $d:=\psi(X)$. Clearly, the graph $G=\operatorname{CG}(X), d$, and the set $\left\{u_{i}\right\}$ uniquely (up to isometry) determine an embedding $X \backslash$ isolated vertices $\}$ in $\mathbb{S}^{2}$.

We obviously have the following constraints for these parameters.

\section{Proposition 3.6}

1. $u_{i}<\pi$ for all $u_{i}$;

2. $u_{i} \geq \alpha(\psi(X))$ for all $u_{i}$, where

$$
\alpha(d):=\cos ^{-1}\left(\frac{\cos d}{1+\cos d}\right)
$$

is the angle of a regular triangle in $\mathbb{S}^{2}$ with sides of length $d$;

3. $\sum_{k \in I(v)} u_{k}=2 \pi$ for all vertices $v$ of $G$, where $I(v)$ is the set of subscripts of angles that are adjacent to $v$.

Let $F$ be a face of $G$. Then $F$ is a polygon with $m$ vertices, where $m=3,4,5$, or 6. Consider all possible cases.

1. $m=3$ : triangle. In this case, $F$ is a regular triangle.

Proposition 3.7 Let $F$ be a triangular face of $G_{13}$ with angles $u_{1}, u_{2}, u_{3}$. Then $u_{1}=$ $u_{2}=u_{3}=\alpha_{13}:=\alpha\left(d_{13}\right)$.

2. $m=4$ : quadrilateral. In this case, $F=A_{1} A_{2} A_{3} A_{4}$ is a rhombus. Then we have $u_{1}=u_{3}$ and $u_{2}=u_{4}$. Using the spherical Pythagorean theorem, one can show that

$$
\cot \frac{u_{1}}{2} \cot \frac{u_{2}}{2}=\cos d \text {. }
$$

Then

$$
u_{2}=\rho\left(u_{1}, d\right):=2 \cot ^{-1}\left(\tan \left(u_{1} / 2\right) \cos d\right) .
$$

Since $u_{2} \geq \alpha(d)$, we have $u_{1}=\rho\left(u_{2}, d\right) \leq \rho(\alpha(d), d)=2 \alpha(d)$ (Fig. 4).

Proposition 3.8 Let $F$ be a quadrilateral of $G_{13}$ with angles $u_{1}, u_{2}, u_{3}$, and $u_{4}$. Then $u_{3}=u_{1}, u_{4}=u_{2}, u_{2}=\rho\left(u_{1}, d_{13}\right), u_{1}=\rho\left(u_{2}, d_{13}\right)$, and $\alpha_{13} \leq u_{i} \leq 2 \alpha_{13}$ for all $i=1,2,3,4$. 
Fig. 4 The graph of the function $u_{2}=\rho\left(u_{1}, d\right)$, where $d=57.1367^{\circ}$

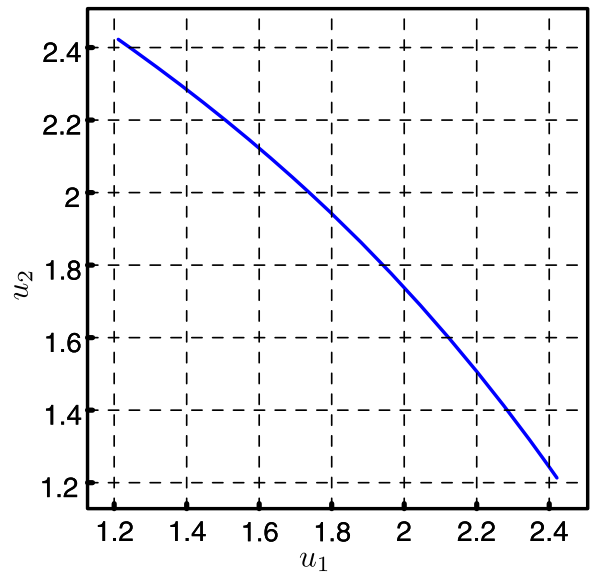

Fig. 5 The set of admissible pairs $\left(u_{1}, u_{2}\right)$ for a pentagon with $d=57.1367^{\circ}$

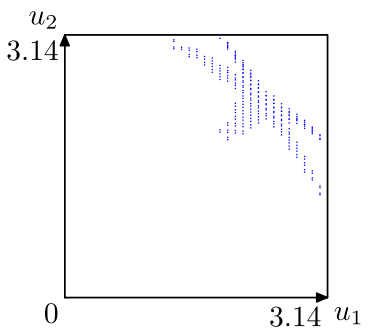

3. $m=5$ : pentagon. In this case, $F$ is a convex equilateral pentagon $A_{1} A_{2} A_{3} A_{4} A_{5}$. Let $u_{1}, u_{2}, u_{3}, u_{4}$, and $u_{5}$ be its angles. Then $F$ is uniquely determined by $d$ and any pair of these angles, for instance, by $\left(u_{1}, u_{2}\right)$ (Fig. 5).

It is not hard, for given parameters $x=u_{1}, y=u_{2}$, and $d$, to find $u_{3}, u_{4}$, and $u_{5}$ as functions of $x, y, d$, i.e. $u_{i}=f_{i}(x, y, d)$, where $i=3$, 4, 5. Let $f_{1}(x, y, d)=x$ and $f_{2}(x, y, d)=y$. Then we have $u_{i}=f_{i}(x, y, d)$ for all $i=1, \ldots, 5$. We find that all $f_{i}(x, y, d) \geq \alpha(d)$.

Denote by $A_{i}^{\prime}$ the image of $A_{i}$ after a Danzer flip. Let $\xi_{i}(x, y, d)$ denote the minimum distance between $A_{i}^{\prime}$ and $A_{j}$, where $j \neq i$. If $F$ is a face of $\operatorname{CG}(X)$ and $\operatorname{CG}(X)$ is irreducible, then $F$ does not admit a Danzer flips. Therefore, $\xi_{i}(x, y, d)<d$ for all $i$. Thus we have the following proposition.

Proposition 3.9 Let $F$ be a pentagonal face of $G_{13}$ with angles $u_{1}, \ldots, u_{5}$. Then $f_{i}\left(u_{1}, u_{2}, d_{13}\right) \geq \alpha_{13}$ and $\xi_{i}\left(u_{1}, u_{2}, d_{13}\right)<d_{13}$ for all $i=1, \ldots, 5$.

4. $m=6$ : hexagon. In this case, $F=A_{1} A_{2} A_{3} A_{4} A_{5} A_{6}$ is a convex equilateral hexagon with angles $u_{1}, \ldots, u_{6}$. Clearly, $F$ is uniquely defined by any three angles and $d$.

Let $u_{i}=g_{i}\left(u_{1}, u_{2}, u_{3}, d\right)$ for $i=4,5,6$. Let $g_{i}\left(u_{1}, u_{2}, u_{3}, d\right)=u_{i}$ for $i=1,2,3$. Then we have $u_{i}=g_{i}\left(u_{1}, u_{2}, u_{3}, d\right)$ for all $i=1, \ldots, 6$. 

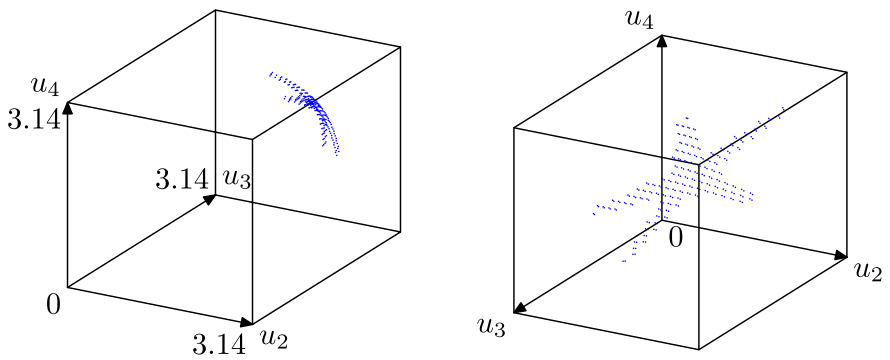

Fig. 6 Admissible angles $\left(u_{1}, u_{2}, u_{3}, u_{4}, u_{5}\right)$ of a pentagon projected into $\left(u_{2}, u_{3}, u_{4}\right)$

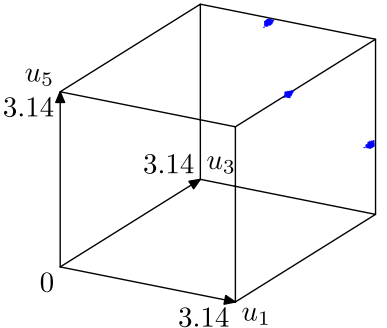

a)

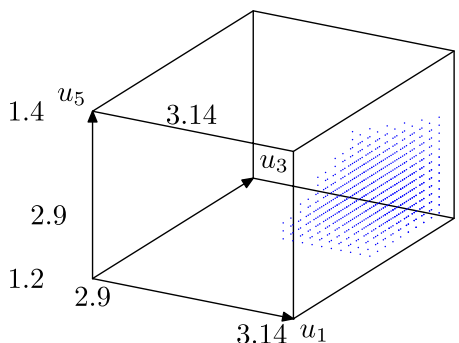

b)

Fig. 7 (a) The set of admissible triplets for $\left(u_{1}, u_{2}, u_{3}\right)$ for empty hexagon with $d=57.1367^{\circ}$. (b) A component with zoom

In fact, for the case $m=6$ we have two subcases: (a) $F$ has no isolated vertices, and (b) $F$ has an isolated vertex.

It is easy to see that for case 4(a) there exists an analog of Proposition 3.9. Let $\zeta_{i}\left(u_{1}, u_{2}, u_{3}, d\right)$ denote the minimum distance between $A_{i}^{\prime}$ and $A_{j}$, where $j \neq i$.

Proposition 3.10 Let $F$ be a hexagonal face of $G_{13}$ with angles $u_{1}, \ldots, u_{6}$. Suppose that the face $F$ has no isolated vertices in its interior. Then $g_{i}\left(u_{1}, u_{2}, u_{3}, d_{13}\right) \geq \alpha_{13}$ and $\zeta_{i}\left(u_{1}, u_{2}, u_{3}, d_{13}\right)<d_{13}$ for all $i=1, \ldots, 6$ (Fig. 7).

Now consider case 4(b). Denote by $\Pi$ the set of all points $p$ in the interior of $F$ such that there is a pair $(i, j), 1 \leq i, j \leq 6, i \neq j$, with $\operatorname{dist}\left(p, A_{i}\right)=\operatorname{dist}\left(p, A_{j}\right)=d$. Clearly, $|\Pi| \leq 18$.

Let $p \in \Pi$ be defined by a pair $(i, j)$. Denote by $K(p)$ the set of all $k=1, \ldots, 6$ such that $k \neq i$ and $k \neq j$. Let

$$
\lambda\left(u_{1}, u_{2}, u_{3}, d\right)=\tilde{\lambda}(F):=\max _{p \in \Pi} \min _{i \in K(p)}\left\{\operatorname{dist}\left(p, A_{i}\right)\right\} .
$$

Since $F$ contains an isolated vertex, we have $\tilde{\lambda}(F) \geq d$.

Proposition 3.11 Let $F$ be a hexagonal face of $G_{13}$ with angles $u_{1}, \ldots, u_{6}$. Suppose that the face $F$ has an isolated vertex in its interior.

Then $g_{i}\left(u_{1}, u_{2}, u_{3}, d_{13}\right) \geq \alpha_{13}$ for all $i=1, \ldots, 6$ and $\lambda\left(u_{1}, u_{2}, u_{3}, d_{13}\right) \geq d_{13}$. 


\section{Proof of Lemma 1}

Here we give a sketch of our computer proof. For more details see http://dcs.isa.ru/ taras/tammes $13 /$.

The proof consists of two parts:

(I) create the list $L_{13}$ of all graphs with 13 vertices that satisfy Corollary 3.1;

(II) using linear approximations and linear programming, remove from the list $L_{13}$ all graphs that do not satisfy the geometric properties of $G_{13}$ (see Propositions 3.6-3.11).

(I). To create $L_{13}$ we use the program plantri (see [24]). ${ }^{2}$ This program is the isomorph-free generator of planar graphs, including triangulations, quadrangulations, and convex polytopes. (Reference [9] describes plantri's principles of operation, the basis for its efficiency, and recursive algorithms behind many of its capabilities.)

The program plantri generates $94,754,965$ graphs in $L_{13}$, i.e. graphs that satisfy Corollary 3.1. Namely, $L_{13}$ contains $30,829,972$ graphs with triangular and quadrilateral faces; 49,665,852 with at least one pentagonal face, and with triangular and quadrilaterals; 13,489,261 with at least one hexagonal face which do not contain isolated vertices; $769,375^{3}$ graphs with one isolated vertex, $505^{3}$ with two isolated vertices, and no graphs with three or more isolated vertices.

(II). Let us consider a graph $G$ from $L_{13}$. We start from the level of approximation $\ell=1$. Now using Propositions 3.6-3.11 we write linear equalities and inequalities for the parameters (angles) $\left\{u_{i}\right\}$ of this graph.

For $\ell=1$ we use the following linear equalities and inequalities:

(i) 13 linear equalities $\sum_{k \in I(v)} u_{k}=2 \pi$ in Proposition 3.6(3);

(ii) since $57.1367^{\circ}=0.9972 \leq d_{13}<1.021=58.5^{\circ}$, we have $1.2113 \leq \alpha_{13}<$ 1.2205;

(iii) for a quadrilateral from Proposition 3.8 we have the equalities $u_{3}=u_{1}, u_{4}=u_{2}$, and the inequalities $\alpha_{13} \leq u_{i} \leq 2 \alpha_{13}, i=1,2$;

(iv) for a quadrilateral, (ii) and $u_{2}=\rho\left(u_{1}, d_{13}\right)$ yield $3.6339 \leq u_{1}+u_{2} \leq 3.779657$;

(v) let $F$ be a pentagonal face. Consider all vectors $U_{5}:=\left\{\left(u_{1}, \ldots, u_{5}\right)\right\}$ that satisfy Proposition 3.9 (see Fig. 6). We use a convex polytope $P_{5}$ in $\mathbb{R}^{5}$ which contains $U_{5}$. Actually, $P_{5}$ is defined by certain linear inequalities. For instance, $2.96 \leq$ $u_{1}+u_{2}-0.63 u_{4} \leq 3.26, u_{1}+u_{3}+1.8 u_{2} \leq 9.05$, etc.

(vi) For a hexagonal face $F$ that contains no isolated vertices, using Proposition 3.10, we find a set of three polytopes $P_{6}^{k}, U_{6} \subset \bigcup_{k=1}^{3} P_{6}^{k}$, which are defined by the inequalities $1.2 \leq u_{k}, u_{k+3} \leq 1.34$ and $2.9 \leq u_{k+1}, u_{k+2}, u_{k+4}, u_{k+5}$;

(vii) for a hexagonal face with an isolated vertex, Proposition 3.11 yields $\sum_{i=1}^{6} u_{i} \geq$ 15.936 .

Using this set of linear inequalities, we find the minimal and maximal value of each variable by linear programming. This gives us a convex region in the space of possible solutions that contains all possible solutions for given graph (if they exist).

\footnotetext{
${ }^{2}$ The authors of this program are Gunnar Brinkmann and Brendan McKay.

${ }^{3}$ This figure may include isomorphic graphs.
} 


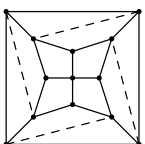

a)

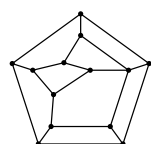

b)

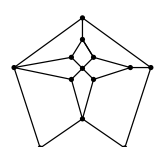

c)

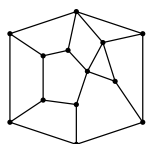

d)

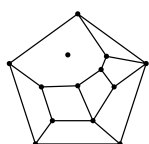

e)

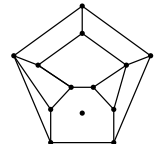

f)

Fig. 8 Strongest eliminated graphs

If the region becomes empty, this means that we can eliminate the graph considered. This step "kills" almost all graphs. After this step, there remain 2013 graphs without hexagons, 40910 graphs with hexagons and without isolated vertices, 9073 graphs with one isolated vertex, and 272 graphs with two isolated vertices.

We use the following idea for $\ell=2$. This region is smaller than the original region, so we can adjust linear estimates for nonlinear equalities and inequalities. For quadrilaterals we adjust inequalities using (iv). For pentagons we use an additional set of inequalities. Namely, using the functions $f_{3}\left(u_{1}, u_{5}, d\right), f_{3}\left(u_{2}, u_{4}, d\right)$, and bounds for $u_{1}, u_{2}, u_{4}, u_{5}, d$, minimal and maximal linear bounds for $u_{3}$.

Repeating this procedure, we obtain a chain of nested convex regions, which contain all possible solutions. This chain converges to an empty or a non-empty region. If this result is empty, the graph is eliminated. After this step, only 260 graphs remain in the main group, 9991 graphs remain in the second group, 126 graphs remain in the third group, and no graphs remain in the fourth group.

For the level of approximation $\ell=3$, we split the region into two smaller regions and repeat the same procedure as for $\ell=2$ independently. For graphs with empty hexagons, we make a specific split by taking different values of $k$ from item (vi) (see above).

Repeating the splitting procedure, we "kill" all graphs except $\Gamma_{13}^{(i)}$.

This result leads to two surprises. We expected that subgraphs were to remain, because they can be infinitesimally close to $\Gamma_{13}$, and so they cannot be eliminated by a computer program. But we did not expect that all other graphs would be killed. Also, we manually found two subgraphs which could be contact graphs: $\Gamma_{13}^{(1)}$ and $\Gamma_{13}^{(2)}$. But we missed the graph $\Gamma_{13}^{(3)}$ with one isolated vertex, which was found by the computer program.

Remark In Fig. 8 are presented examples of graphs which are not isomorphic to $\Gamma_{13}^{(i)}$ and have been eliminated only after many iterations. The strongest of the graphs in Fig. 8 is (a). This graph is also a subgraph of $\Gamma_{13}^{(0)}$. After eliminating four edges, the graph contains four pentagons. The reason why it was eliminated is that there are angles $u_{i}$ which are slightly larger than $\pi$, so that the pentagons are not convex. Therefore, this graph is not irreducible. Most other surviving graphs were "strong" because they have several pentagons and hexagons. Note that here we use weak bounds for pentagons and hexagons given by (v), (vi), (vii). Our elimination procedure works very fast when we have sufficiently many triangles and quadrilaterals, and it works worse (slowly) when we have several pentagons and hexagons. 


\section{Proof of Lemma 2}

Proof This proof is based on geometric properties of $G_{13}$. In Sect. 4 we substitute all nonlinear equations by certain linear inequalities. Note that the statement $d_{13} \approx \delta_{13}$ is a by-product of this approximation. Here we prove that $d_{13}=\delta_{13}$ based on the original equations.

Lemma 1 says that $G_{13}=\Gamma_{13}^{(i)}$, where $i=0,1,2$, or 3 . We are going to prove that if $\operatorname{CG}(X)=\Gamma_{13}^{(i)}$ with $i>0$, then $\psi(X)<\delta_{13}=\psi\left(P_{13}\right)$.

5.0. Angles of $\Gamma_{13}^{(2)} \quad$ Let $u_{0}:=\alpha(d)$. For $G_{13}=\Gamma_{13}^{(2)}$ we have (see Fig. 9):

$$
\begin{gathered}
u_{5}=\rho\left(u_{1}, d\right) \quad u_{6}=\rho\left(u_{2}, d\right) \quad u_{9}=2 \pi-u_{5}-u_{6} \\
u_{13}=\rho\left(u_{9}, d\right) \quad u_{14}=2 \pi-u_{0}-u_{13}-u_{2} \quad u_{10}=\rho\left(u_{14}\right) \\
u_{7}=2 \pi-u_{6}-u_{10} \quad u_{3}=\rho\left(u_{7}, d\right) \quad u_{4}=2 \pi-u_{1}-u_{2}-u_{3} \\
u_{8}=\rho\left(u_{4}, d\right) \quad u_{11}=2 \pi-u_{7}-u_{8} \quad u_{12}=2 \pi-u_{8}-u_{5} \\
u_{15}=\rho\left(u_{11}, d\right) \quad u_{16}=\rho\left(u_{12}, d\right)
\end{gathered}
$$

Therefore, for $3 \leq i \leq 16$ the value $u_{i}$ are functions of the variables $u_{1}, u_{2}$, and $d$. Since we have also an additional equation for the vertex $v_{8}$ (see Figs. 1 and 9):

$$
u_{0}+u_{15}+u_{4}+u_{16}=2 \pi,
$$

the value $d$ is a function of $u_{1}, u_{2}$; also, $u_{2}$ is a function of the variables $u_{1}$ and $d$. Thus, all $u_{i}$ and $d$ are functions of $u_{1}, u_{2}$ or of $u_{1}, d$.

Now we consider three cases $G_{13}=\Gamma_{13}^{(i)}$, where $i=1,2,3$.

5.1. The case $G_{13}=\Gamma_{13}^{(1)}$ In this case $u_{17}=u_{0}$. Then for vertex $v_{7}$ we have the equation

$$
u_{1}+u_{13}+u_{0}+u_{16}=2 \pi .
$$

From this it follows that $u_{1}$ and, therefore, all $u_{i}$ are functions of $d$. Note that

$$
u_{18}=2 \pi-u_{14}-u_{3}-u_{15} .
$$

Fig. 9 Angles of $\Gamma_{13}^{(2)}$

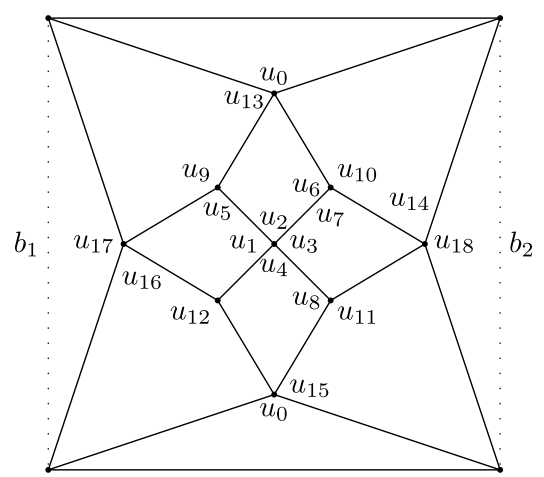


Fig. 10 The graph of the function $u_{18}(d)$

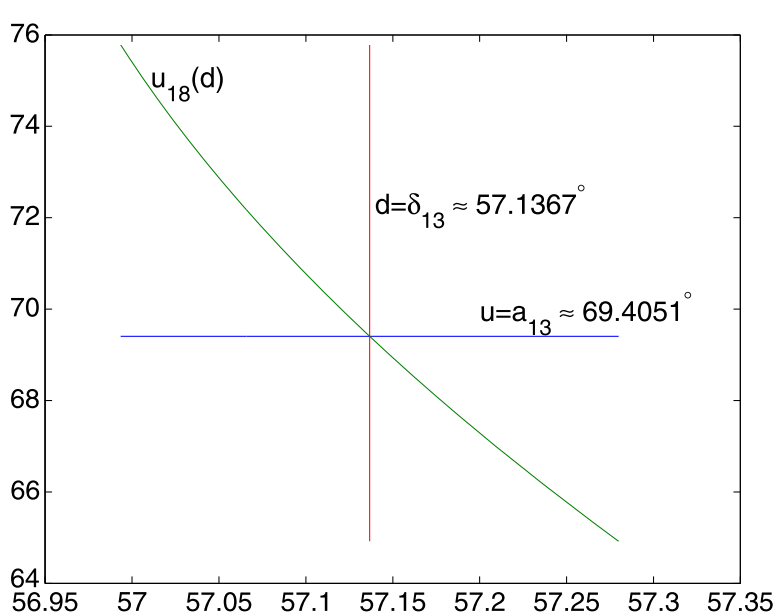

Fig. $11 D_{1}$ and $D_{2}$

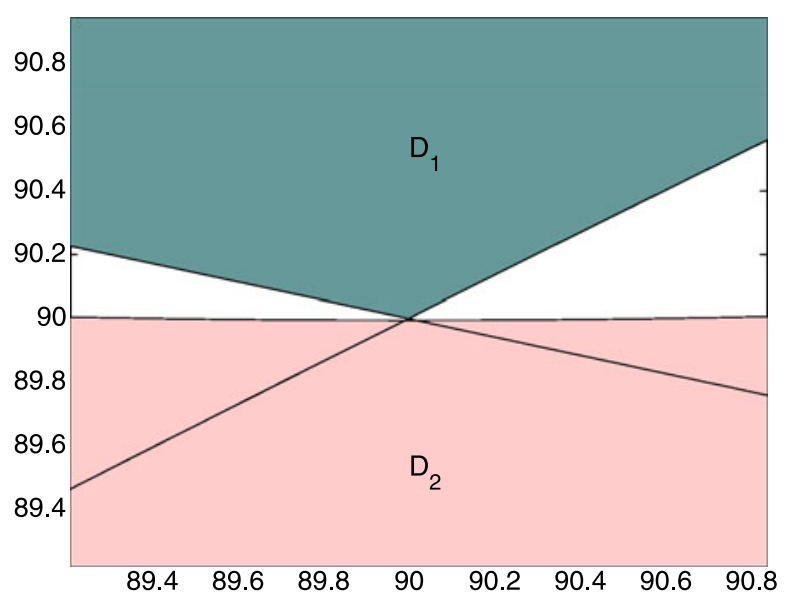

Thus, $u_{18}$ is a function of $d$ (see Fig. 10).

If $G_{13}=\Gamma_{13}^{(1)}$, then $u_{18}>u_{0} \geq a_{13}$. Since the function $u_{18}(d)$ is monotonous decreasing, we have $u_{18}(d)>a_{13}$ only if $d<\delta_{13}$. Thus, $G_{13} \neq \Gamma_{13}^{(1)}$.

5.2. The case $G_{13}=\Gamma_{13}^{(2)}$ It is already shown that $d$ and all $u_{i}$ are functions of $u_{1}, u_{2}$. Let

$$
\begin{aligned}
& D_{1}:=\left\{\left(u_{1}, u_{2}\right): u_{17} \geq u_{0}, u_{18} \geq u_{0}\right\} \quad \text { and } \\
& D_{2}:=\left\{\left(u_{1}, u_{2}\right): u_{0}=\alpha(d) \geq a_{13}\right\} .
\end{aligned}
$$

We can see from Fig. 11 that the intersection $I:=D_{1} \cap D_{2} \subset \mathbb{R}^{2}$ consists of one point with $u_{1}=u_{2}=90^{\circ}$. It is not hard to prove this fact. Indeed, conversely, $d_{13}>\delta_{13}$, and there is a point $\left(u_{1}, u_{2}\right)$ on the boundary of $I$ such that $u_{17}=u_{0}$ or $u_{18}=u_{0}$. Therefore, we have the same case as in case 5.1, a contradiction. Thus, $G_{13} \neq \Gamma_{13}^{(2)}$. 

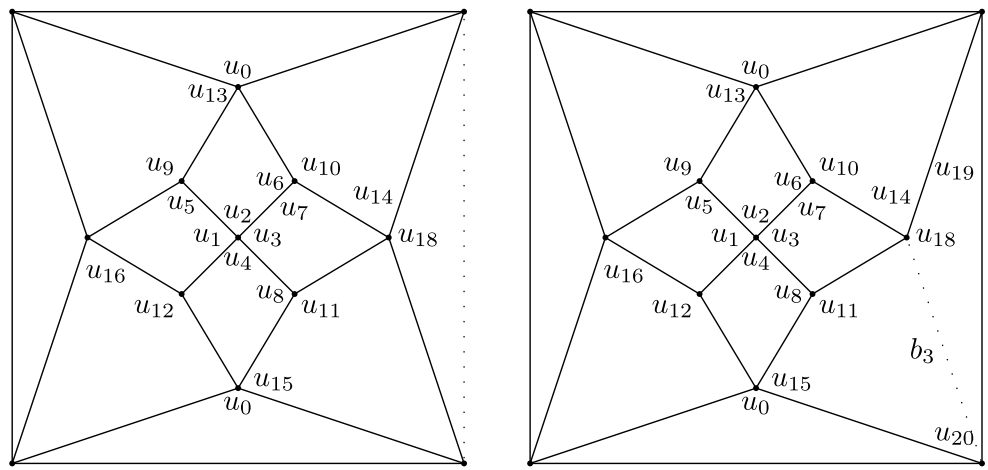

Fig. 12 Two subcases for the case $G_{13}=\Gamma_{13}^{(3)}$

5.3. The case $G_{13}=\Gamma_{13}^{(3)}$ This case can be considered by the same method as the case $G_{13}=\Gamma_{13}^{(2)}$. Actually, for given $u_{1}, u_{2}$, and $d$, all angles $u_{i}, 3 \leq i \leq 16, i \neq 15$ can be found by the same formulas as in case 5.0. On the other hand,

$$
u_{15}=2 \pi-u_{4}-u_{16}-u_{0} .
$$

Then all $u_{i}$ are functions of the variables $u_{1}, u_{2}$, and $d$. Since $u_{17}=u_{0}$ (or equivalently $b_{1}=d$ ), we have the equation

$$
u_{1}+u_{13}+u_{0}+u_{16}=2 \pi .
$$

We find that all $u_{i}$ depend on two parameters.

The vertex $v_{13}$ is isolated. In fact, we can shift this point in such a way that at least two edges $v_{13} v_{k}$, where $k=8,9,10,12$, have lengths $d$. Then for two other edges we have the inequalities $\operatorname{dist}\left(v_{13}, v_{i}\right) \geq d$ and $\operatorname{dist}\left(v_{13}, v_{j}\right) \geq d$.

Arguing as in case 5.2, we can show that there are parameters $u_{1}, u_{2}$ such that $u_{0}>a_{13}$ and at least one of the inequalities $\operatorname{dist}\left(v_{13}, v_{k}\right) \geq d, k=i, j$, becomes an equality. It is not hard to see that there are exactly two geometrically nonequivalent cases with exactly one edge $v_{13} v_{k}, k=8,9,10$, or 12 , such that $\operatorname{dist}\left(v_{13} v_{k}\right)>d$. These cases are shown in Fig. 12.

Actually, the first subcase is case 5.1. For the second subcase consider the pentagon $F:=v_{5} v_{8} v_{12} v_{13} v_{10}$. All angles of $F$ can be found as functions of $u_{1}, d$. Since $d$ and any two angles of $F$ define all other angles, we can use one of these equations to find $u_{1}$ as a function of $d$. Then $u_{19}$ (see Fig. 12) is a function of $d$. In fact, the graph of the function $u_{19}(d)$ is very similar to the graph $u_{18}(d)$ in Fig. 10, and $u_{19}(d)$ is a monotonous decreasing function. Thus, $u_{19}(d)$ cannot be greater than $a_{13}$, and $G_{13} \neq \Gamma_{13}^{(3)}$.

We see that if $\mathrm{CG}(X)=G_{13}$, then $\mathrm{CG}(X)$ is isomorphic to $\Gamma_{13}$. Moreover, $X$ is uniquely defined up to isometry and $\psi(X)=\delta_{13} \approx 57.1367^{\circ}$. This completes the proof.

Acknowledgements We wish to thank Robert Connelly, Alexey Glazyrin, Nikita Netsvetaev, and Günter Ziegler for helpful discussions and comments. Supported by the Russian government project 11.G34.31.0053. 
O.R. Musin's research is supported in part by NSF grant DMS-0807640 and NSA grant MSPF-08G201. A.S. Tarasov's research is supported by program P15 of presidium of RAS and RFBR grant 11-0100633 and 11-01-00735.

\section{References}

1. Aigner, M., Ziegler, G.M.: Proofs from THE BOOK. Springer, Berlin (1998) 1st edn. and (2002) 2nd edn.

2. Anstreicher, K.: The thirteen spheres: A new proof. Discrete Comput. Geom. 31, 613-625 (2004)

3. Bachoc, C., Vallentin, F.: New upper bounds for kissing numbers from semidefinite programming. J. Am. Math. Soc. 21, 909-924 (2008)

4. Böröczky, K.: The problem of Tammes for $n=11$. Studia Sci. Math. Hung. 18, 165-171 (1983)

5. Böröczky, K.: The Newton-Gregory problem revisited. In: Bezdek, A. (ed.) Discrete Geometry, pp. 103-110. Dekker, New York (2003)

6. Böröczky, K., Szabó, L.: Arrangements of 13 points on a sphere. In: Bezdek, A. (ed.) Discrete Geometry, pp. 111-184. Dekker, New York (2003)

7. Böröczky, K., Szabó, L.: Arrangements of 14, 15, 16 and 17 points on a sphere. Studia Sci. Math. Hung. 40, 407-421 (2003)

8. Brass, P., Moser, W.O.J., Pach, J.: Research Problems in Discrete Geometry. Springer, Berlin (2005)

9. Brinkmann, G., McKay, B.D.: Fast generation of planar graphs (expanded edition). http://cs.anu. edu.au/ bdm/papers/plantri-full.pdf

10. Casselman, B.: The difficulties of kissing in three dimensions. Not. Am. Math. Soc. 51, 884-885 (2004)

11. Danzer, L.: Finite point-sets on $\mathbf{S}^{2}$ with minimum distance as large as possible. Discrete Math. 60, 3-66 (1986)

12. Fejes Tóth, L.: Über die Abschätzung des kürzesten Abstandes zweier Punkte eines auf einer Kugelfläche liegenden Punktsystems. Jber. Deutch. Math. Verein. 53, 66-68 (1943)

13. Fejes Tóth, L.: Lagerungen in der Ebene, auf der Kugel und in Raum. Springer, Berlin (1953). Russian translation, Moscow, 1958

14. Hales, T.: The status of the Kepler conjecture. Math. Intell. 16, 47-58 (1994)

15. Hoppe, R.: Bemerkung der Redaktion. Archiv Math. Phys. (Grunet) 56, 307-312 (1874)

16. Hsiang, W.-Y.: Least Action Principle of Crystal Formation of Dense Packing Type and Kepler's Conjecture. World Scientific, Singapore (2001)

17. Leech, J.: The problem of the thirteen spheres. Math. Gaz. 41, 22-23 (1956)

18. Levenshtein, V.I.: On bounds for packing in $n$-dimensional Euclidean space. Sov. Math. Dokl. 20(2), 417-421 (1979)

19. Maehara, H.: Isoperimetric theorem for spherical polygons and the problem of 13 spheres. Ryukyu Math. J. 14, 41-57 (2001)

20. Maehara, H.: The problem of thirteen spheres—a proof for undergraduates. Eur. J. Comb. 28, 17701778 (2007)

21. Musin, O.R.: The kissing problem in three dimensions. Discrete Comput. Geom. 35, 375-384 (2006)

22. Musin, O.R.: The kissing number in four dimensions. Ann. Math. 168, 1-32 (2008)

23. Odlyzko, A.M., Sloane, N.J.A.: New bounds on the number of unit spheres that can touch a unit sphere in $n$ dimensions. J. Comb. Theory, Ser. A 26, 210-214 (1979)

24. Plantri, Fullgen: http://cs.anu.edu.au/ bdm/plantri/

25. Pfender, F., Ziegler, G.M.: Kissing numbers, sphere packings, and some unexpected proofs. Not. Am. Math. Soc. 51, 873-883 (2004)

26. Robinson, R.M.: Arrangement of 24 circles on a sphere. Math. Ann. 144, 17-48 (1961)

27. Schütte, K., van der Waerden, B.L.: Auf welcher Kugel haben 5, 6, 7, 8 oder 9 Punkte mit Mindestabstand 1 Platz? Math. Ann. 123, 96-124 (1951)

28. Schütte, K., van der Waerden, B.L.: Das Problem der dreizehn Kugeln. Math. Ann. 125, 325-334 (1953)

29. Szpiro, G.G.: Kepler's Conjecture. Wiley, New York (2002)

30. Tammes, R.M.L.: On the origin number and arrangement of the places of exits on the surface of pollengrains. Rec. Trv. Bot. Neerl. 27, 1-84 (1930) 